\title{
CUTANEOUS POLYARTERITIS NODOSA AND TUBERCULOSIS IN A CHILD: A VERY RARE CASE REPORT
}

Reinan Tavares Campos ${ }^{1, \star}$, Lucia Maria Mattei Arruda Campos ${ }^{1}$, Clovis Arthur Almeida da Silva ${ }^{1}$, Pedro Vale Bede ${ }^{1}$, Maria Fernanda Badue Pereira ${ }^{1}$, Cinthia Moreno Garcia ${ }^{1}$, Maíra Oliveira Moraes ${ }^{1}$

1.Universidade de São Paulo, São Paulo (SP), Brazil.

*Corresponding author: reinantavares@gmail.com

\section{BACKGROUND}

Polyarteritis nodosa (PAN) is a rare necrotizing vasculitis of medium and small size arteries. This primary vasculitis in pediatric populations may be triggered by infectious, mainly caused by Streptococcus. There are very few cases reported associated with tuberculosis, especially in adults. Therefore, we reported here a case of PAN possibly triggered after pulmonary tuberculosis.

\section{CASE REPORT}

A 5-year-old boy presented to the emergency care of our university hospital with a history of daily fever associated with intermittent abdominal pain, headache and weight loss for the previous 20 days. In the last 10 days, he had limb pain. At admission, erythematous, painful, nonulcerated deep nodule lesions $(1-1.5 \mathrm{~cm})$ were observed in lower limbs. At that moment, laboratory exams showed hemoglobin $10.4 \mathrm{~g} / \mathrm{dL}$, white cells $28.060 / \mathrm{mm}^{3}$, platelets $670 \times 10^{3} / \mu \mathrm{L}$ and C-reactive protein $154.58 \mathrm{mg} / \mathrm{dL}$. Renal and hepatic tests, and abdominal ultrasound were normal. Antistreptolysin $\mathrm{O}$ and antineutrophil cytoplasmic autoantibodies (ANCA) were negative. Serological test and polymerase chain reaction for COVID-19, and serology for HIV and other infections were systematically negative. Acid-fast bacilli and molecular test for Mycobacterium tuberculosis were negative on three samples of gastric aspirate. However, tuberculin skin test was positive $(15 \mathrm{~mm})$, and computed tomography angiography revealed hypoattenuating and enlarged lymph nodes in the left subcarinal and hilar chains associated with consolidating band opacity in the lower left lung lobe, predominantly atelectatic, indicating primary tuberculosis. A skin lesion biopsy demonstrated lymphohistiocytic infiltrate in medium size arteries, suggestive of polyarteritis nodosa. Therefore, cutaneous polyarteritis nodosa associated to tuberculosis infection was diagnosed, and steroids and tuberculosis treatment was started, with complete improvement of symptoms.

\section{CONCLUSION}

To our knowledge, this was the first case report of cutaneous PAN and tuberculosis in a child. This fact highlights the importance of the anatomopathological study in panniculitis and alerts to the possibility of an association between $M$. tuberculosis infection and PAN.

\section{KEYWORDS}

Polyarteritis nodosa, Pediatric, Tuberculosis. 